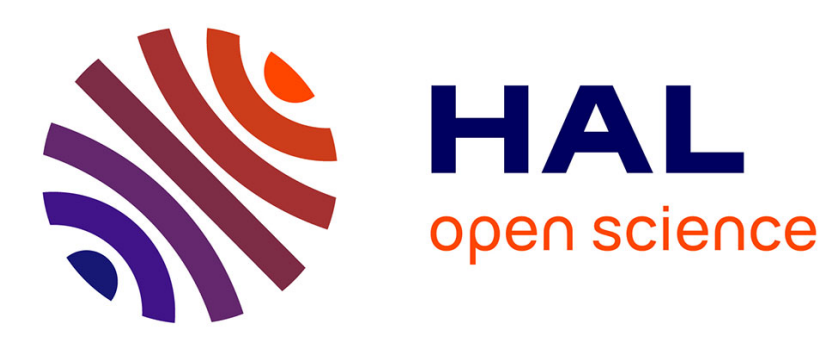

\title{
Erosion onset of a cohesionless granular medium by an immersed impinging round jet
}

\author{
F. Brunier-Coulin, P. Cuéllar, P. Philippe
}

\section{To cite this version:}

F. Brunier-Coulin, P. Cuéllar, P. Philippe. Erosion onset of a cohesionless granular medium by an immersed impinging round jet. Physical Review Fluids, 2017, 2 (03), pp.034302. hal-01734789

\author{
HAL Id: hal-01734789 \\ https://hal.science/hal-01734789
}

Submitted on 15 Mar 2018

HAL is a multi-disciplinary open access archive for the deposit and dissemination of scientific research documents, whether they are published or not. The documents may come from teaching and research institutions in France or abroad, or from public or private research centers.
L'archive ouverte pluridisciplinaire HAL, est destinée au dépôt et à la diffusion de documents scientifiques de niveau recherche, publiés ou non, émanant des établissements d'enseignement et de recherche français ou étrangers, des laboratoires publics ou privés. 


\title{
Erosion onset of a cohesionless granular medium by an immersed impinging round jet
}

\author{
Florian Brunier-Coulin, ${ }^{1}$ Pablo Cuéllar, ${ }^{1}$ and Pierre Philippe ${ }^{1}$ \\ ${ }^{1}$ IRSTEA, UR RECOVER, 3275 route de Cézanne, \\ CS 40061, Aix-en-Provence, F-13182 France
}

(Dated: February 3, 2017)

\begin{abstract}
Among different devices developed quite recently to quantify the resistance to erosion of a natural soil within the broader context of dyke safety, the most commonly used is probably the Jet Erosion Test (JET) in which a scouring crater is induced by impingement of an immersed water jet. A comprehensive experimental investigation on the jet erosion in the specific situation of a cohesionless granular material is presented here. The tests were performed combining special optical techniques allowing for an accurate measurement of the scouring onset and evolution inside an artificially translucent granular sample. The impinging jet hydrodynamics are also analyzed empirically validating the use of a self-similar theoretical framework for the laminar round jet. The critical conditions at the onset of erosion appear to be best described by a dimensionless Shields number based on the inertial drag force created by the fluid flow on the eroded particles rather than on the pressure gradients around them. To conclude, a tentative empirical model for the maximal flow velocity initiating erosion at the bottom of the scoured crater is put forward and discussed in the light of some preliminary results.
\end{abstract}


Author-produced version of the article published in Physical Review Fluids, 2017, 2, 034302.

The original publication is available at https://journals.aps.org/prfluids/abstract/10.1103/PhysRevFluids.2.034302

DOI: 10.1103/PhysRevFluids.2.034302

\section{INTRODUCTION}

The erosion and transport of sediments is a key problem for many fields, both in nature and industry, such as in the coastal engineering regarding the prediction of estuary and river bed evolution $[21,35]$, or in the civil engineering concerning the safety of embankment dams and dikes $[12,13]$. Within this latter context of great social relevance, there are statistical estimates [22] showing that surface erosion, which generally results from an overflowing of the earthwork, causes about $50 \%$ of the embankment dam and levee failures whereas a slightly smaller proportion can be attributed to internal erosion induced by water infiltration through the embankment or its foundation. In order to enhance the erosion resistance of the earthen hydraulic structures, it appears therefore essential to improve in the first place our knowledge about the elementary mechanisms involved in the erosion processes at the surface of a soil sample. Basically, the surface erosion of a soil is the process by which elementary soil particles are detached under the action of a superficial water flow that generates hydrodynamic stresses at the interface of the soil, which are related to fluid pressure and velocity gradients. This apparently simple situation is complicated both by the type of soil, which can be either cohesive or cohesionless with a wide range of particle sizes and shapes, and by the fluid flow itself whose regime is most commonly turbulent or transitional, involving large stress fluctuations. Practically, and regardless the type of soil, the resistance to erosion can be in principle defined by a critical shear stress $\tau_{c}$ above which the hydrodynamical shear stress $\tau_{s}$ generated by the water flow is large enough to dislodge particles at the soil's surface. Obviously, this critical threshold is highly dependent on the mutual interactions between particles, which differ considerably depending on whether the soil is cohesionless or cohesive. In this latter case, a second soil parameter $k$ can be introduced to account for the kinetics of erosion once the critical threshold is exceeded, for instance considering that the rate of erosion can be assumed simply proportional to the excess shear stress $\tau_{s}-\tau_{c}[3,36]$.

Both erosion parameters $\tau_{c}$ and $k$ are generally regarded as inherent properties of the material, and can be evaluated for natural soils by means of specific erosion tests like the Hole Erosion Test [10], the Erosion Function Apparatus [14], or the annular flume [39]. However, one of the devices most widely used for this purpose is the Jet Erosion Test (JET) described in detail by Hanson and Cook [24] partly due to its ability to perform experiments in situ. A 
Jet Erosion Test consists in applying an immersed round jet perpendicularly to the surface of a soil sample and measuring the scouring depth increase with time. Then, it is possible to estimate the two erosion parameters $\tau_{c}$ and $k$ for instance by fitting a very simplified model of interpretation to the experimental data [24]. It can be noted that this model was derived from strong assumptions on the fluid flow which speculate a straightforward link to a free jet situation and its related self-similar model [41], giving little or none consideration to the jet deflection by the soil surface or to the reverse flow created inside the scour crater [30, 31]. Some improvements of the model have already been achieved from both experimental and analytical results for the case of a jet impinging on a wall $[8,9,23,26,37]$, but still without considering the scouring crater development and its subsequent implications for the fluid flow at the soil surface, which rapidly departs from the plane conditions. So far, the extent to which these assumptions are relevant in order to describe properly the actual complex hydrodynamical configuration is still unclear. It can also be noted that all these previous works have dealt with turbulent jets out of either water or air, whereas the present study relies on experimental results based on specific optical techniques which involve a liquid significantly more viscous than water, and focuses exclusively on laminar jets where the jet Reynolds numbers are at least two orders of magnitude lower. Another advantage of the Jet Erosion Test concerns its ability to analyze different kinds of soil, both cohesive and cohesionless ones. The present study is restricted to the particular case of a cohesionless granular material, for which several previous works have been carried out in the past. Most of the available references focus on the morphology of the scour crater, with the eroding fluid being either a liquid $[1,25,26,30]$ or a gas $[6,17,27,28,32]$, the latter mainly motivated by the issue of a retrograde rocket landing on an extraterrestrial planet. In contrast, the present study disregards the crater morphology and concentrates solely on the onset of granular erosion under the action of an immersed impinging liquid jet, extending the recent results by Badr et al. [5] obtained for a plane jet to the most usual 3D configuration of a round jet, and proposing an empirical model for erosion threshold inside the scouring crater. As demonstrated by Badr et al. [5], the characteristical hydrodynamic quantities of the fluid flow can be satisfactorily and consistently estimated from the classical self-similar free jet models, provided that a careful analysis is implemented, which in particular includes the introduction of a virtual origin as will be discussed more in depth in section III. Then, the hydrodynamical condition for particle dislodgement at the surface of the granular bed 
is classically quantified by the dimensionless Shields number $S h$ which compares the flow shear stress $\tau_{s}$ to the buoyant weight of the soil particles [15]. Note that for a turbulent impinging jet Sutherland and Dalziel [42] found that the onset for crater formation could be best described in terms of the Rouse number. But this number that is specifically defined for fully turbulent flow regimes is no more relevant for a laminar jet as will be studied here after. Several previous studies have focused on the onset of particle motion in laminar flow condition with irregular granular substrates [16, 18, 29, 34] or also regular ones [2]. Based on their results considering a planar impinging liquid jet, Badr et al. [5] have concluded that the initiation of grain movement can be satisfactorily predicted by a critical inertial Shields number around 1 for particle Reynolds numbers ranging from 3 to 80, by assuming a simplified expression for the inertial flow stress derived from both laminar and turbulent free plane jet models [41] which therefore seem to be valid for a jet impinging on a granular sample. The present study provides an experimental contribution to a better understanding of the granular erosion mechanisms and most specifically to the definition of a critical inertial Shields number, based on a parametric analysis using artificial soils subjected to an impinging round jet. Both the optical techniques and the employed model materials, made out of glass beads immersed in an oil mixture, are described in section II along with the experimental set-up and the testing protocols that have been followed. Furthermore, a set of complementary experiments featuring a digital post-processing by means of the Particle Image Velocimetry (PIV) provide then the basis for a detailed analysis of the hydrodynamics of the impinging jet shown in section III and its comparison with the laminar self-similar theoretical model originally proposed by Schlichting [41]. Finally, section IV is devoted to an analysis of the experimental results: Firstly, two alternative definitions for the critical inertial Shields number referring to the free round jet model obtained in the previous section are examined and discussed, leading to the adoption of a final critical value fully consistent with Badr's results [5]; Then, a tentative empirical model accounting for the fluid velocity reduction inside the crater scoured in the granular sample is presented and some of its limitations are outlined. 
Author-produced version of the article published in Physical Review Fluids, 2017, 2, 034302.

The original publication is available at https://journals.aps.org/prfluids/abstract/10.1103/PhysRevFluids.2.034302 DOI: 10.1103/PhysRevFluids.2.034302

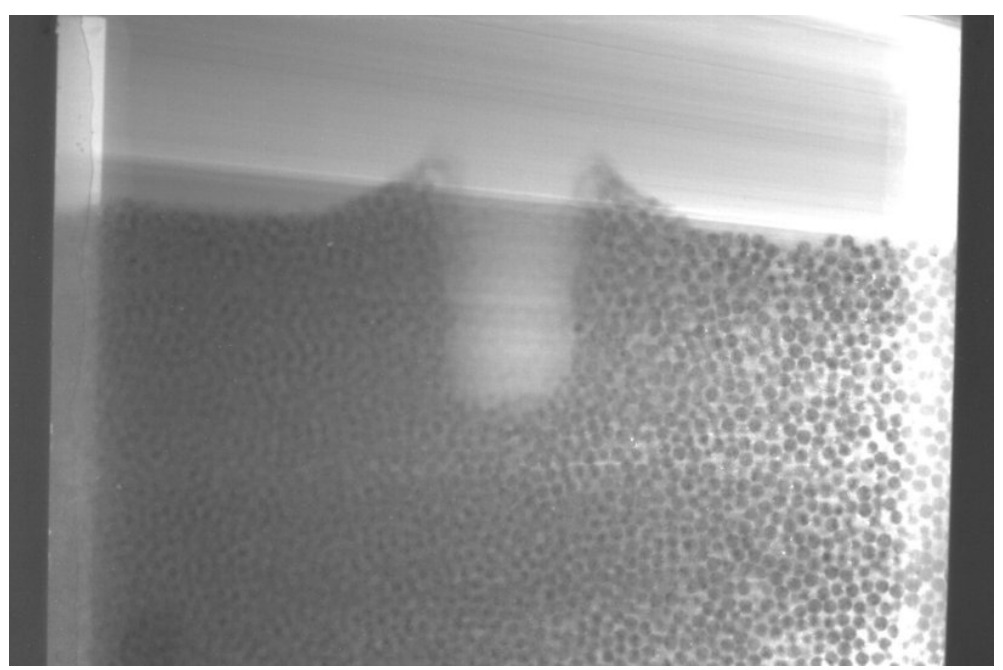

FIG. 1. Typical image of a scour crater within a bead sample $(d=3 \mathrm{~mm})$ visualized by laser illumination in a plane containing the jet axis during a Jet Erosion Test.

\section{EXPERIMENTS}

\section{A. Materials and optical techniques}

This study focuses on the processes of erosion and crater scouring in a granular sample subject to the solicitation imposed by an impinging jet. In order to achieve a direct internal observation of the granular medium without perturbing it, the optical techniques of Refractive Index Matching (RIM) along with the Planar Laser Induced Fluorescence (PLIF) were used during the tests. These techniques, which are becoming quite popular among fluid mechanics experiments as, for instance, in some previous studies of the authors [7, 38], will be only briefly described. Further details can be found for instance in [19]. The RIM technique consists in using solid particles that have the same refractive index as the liquid in order to make the interface between the two phases almost undistinguishable. Inside this transparent medium it is then possible to visualize a chosen $2 \mathrm{D}$ region by illuminating the desired area with a planar laser after prior addition of a fluorescent dye to the liquid. The laser excites the dye and makes the fluid fluoresce at a higher wavelength than the incident light. By using a specifically selected high pass optical filter, the scattered light can be separated from the fluoresced light and the liquid is consequently bright in the resulting image while the particles remain dark. A typical image obtained with this technique is shown in Figure 1. Here we chose to use a granular phase made out of spherical borosilicate glass 


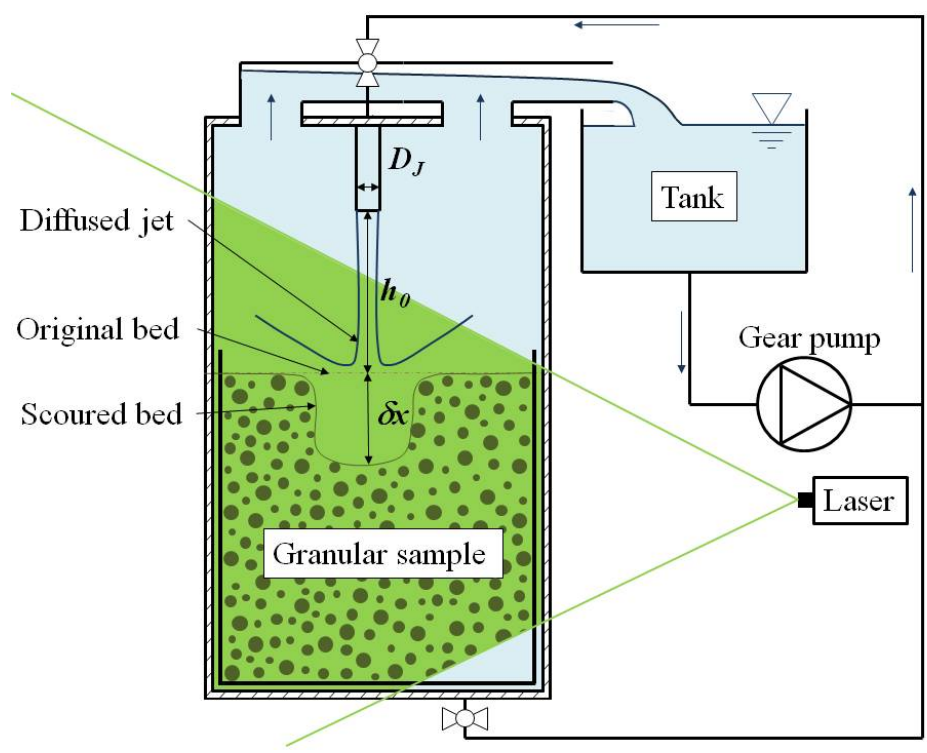

FIG. 2. Sketch of the adapted Jet Erosion Test device for index-matching conditions.

beads and a liquid which is a mixture of two mineral oils: light mineral oil (supplied by Sigma-Aldrich) and immersion oil type A (supplied by Cargille), with mass fractions of $90 \%$ and $10 \%$ respectively. The matched refractive index of the oil mixture is $n=1.472$ at $23^{\circ} \mathrm{C}$ as measured by a refractometer (Master-RI provided by Atago). Its density was estimated to $\rho=846 \mathrm{~kg} \cdot \mathrm{m}^{-3}$ from mass fractions and suppliers' data and its dynamical viscosity was measured to $\mu=28 \pm 2 c P$ at $23^{\circ} \mathrm{c}$ with a Falling Ball Viscometer (supplied by Gilmont). The kinematic viscosity is therefore approximately $\nu=(33 \pm 3) \times 10^{-6} \mathrm{~m}^{2} . \mathrm{s}^{-1}$. The density of the borosilicate glass beads is $\rho_{g}=2230 \mathrm{~kg} \cdot \mathrm{m}^{-3}$ and the different bead diameters used in this study are $d=1.0 \pm 0.1 \mathrm{~mm}, d=3.0 \pm 0.2 \mathrm{~mm}, d=5.0 \pm 0.2 \mathrm{~mm}$, and $d=7.0 \pm 0.3 \mathrm{~mm}$. The $1 \mathrm{~mm}$-beads were supplied by Sigma-Aldrich while the other ones were manufactured by SiLi Beads (type M, matt).

\section{B. Adapted JET device and sample preparation}

The classical JET device has been adapted to fulfill the requirements of the optical techniques implemented in this study. A sketch of the set-up is presented in Figure 2. The cell is a rectangular box with cross-section area $10 \times 20 \mathrm{~cm}^{2}$ and total available height equal to $40 \mathrm{~cm}$. The upper side is removable and includes two outlets, used to create an overflow system, and a central inlet, directly connected to a gear pump (VG 540 supplied by Verder). 
A metal tube composed of one to four screw-fitting sections of individual length $4 \mathrm{~cm}$ is mounted at this inlet to generate a downward vertical immersed jet at a controlled flow rate supplied by the pump from a liquid reservoir tank. The overflow system at the top of the box brings the liquid back to the reservoir in a closed pipe circuit. A planar laser (532nm, 100mW, provided by Coherent) with a fan angle of $60^{\circ}$ and a uniform angular intensity distribution is placed precisely in order to illuminate the median vertical plane of the cell as shown in Figure 2. Red Nile dye (supplied by Fluka) is used as a convenient fluorescent dye, miscible in our oil mixture and adapted to the $532 \mathrm{~nm}$ laser wavelength, together with a high pass optical filter at $580 \mathrm{~nm}$. The images were mostly recorded by a digital camera (MQ042MG-CM supplied by Ximea) with a pixel resolution of $2048 \times 2048$ and a frame rate up to 20 images per second. A fast camera (see details further on in section II C) was specifically utilized for the PIV experiments to reach much higher frame rates. The preparation of the granular sample was performed as follows. A given mass of beads is firstly poured into the cell and afterwards the pump is used to supply the liquid through a second parallel path connected to the bottom of the cell, with a very slow flow rate. Once the granular sample is entirely immersed, the pump is stopped and the bead sample is manually stirred with moderate force by means of a long metal rod in order to obtain an almost reproducible packing, with a solid volume fraction of approximately $0.61 \pm 0.01$ whatever the bead diameter, and also to get rid of any air bubbles trapped during the submersion phase. The rod is pulled out of the bed nearby a corner of the cell to avoid damaging the flatness of the granular bed which is controlled visually during and after stirring, once the planar laser is turned on. Then, the top side of the cell is set back and tightened with screws, and some more liquid is pumped in with a slightly higher flow rate until the overflow system is reached. At this stage, the pump is momentarily stopped in order to allow the closure and opening of the filling and jet vanes respectively. The set-up is this way ready for launching the impinging liquid jet. The beads are kept in place until all experiments for a fixed diameter $d$ have been carried out but the stirring procedure is operated before each experiment. The initial position of the bed surface level is derived by image processing and more precisely from a vertical grey scale profile calculated on a thresholded image corresponding to a selected region of interest that is centered on the jetaxis and has a width equal to the injection diameter $D_{J}$. The shape of the profile is roughly a step, from lighter liquid area to darker granular medium, and the mid-height position is 
used to define the top surface level.

Besides the bead diameter $d$, the other experimental parameters that were varied are the diameter $D_{J}$ of the jet injection tube and the initial distance $h_{0}$ from the jet nozzle to the granular bed surface, which was varied depending on both the mass of beads in the sample and on the jet tube length that ranged from $4 \mathrm{~cm}$ (only one tube section) to $16 \mathrm{~cm}$ (all four tube sections). Using an expandable length for the injection tube guarantees to keep roughly the same bed height, even for small initial distance $h_{0}$. Note that a negligible impact of the injection length on the jet flow is expected as Reynolds numbers are less 230 and diameter reduction with the inlet and outlet pipes (internal diameter around $20 \mathrm{~mm}$ ) remains rather small. Finally, a direct control of the erosion and scouring processes of interest here is obtained by varying the jet flow rate $Q_{J}$ and the corresponding mean jet velocity $U_{J}=\frac{4 Q_{J}}{\pi D_{J}{ }^{2}}$ through a change of the pump motor frequency and using a predetermined linear calibration curve. The maximal achievable flow rate supplied by our pump was about $230 \mathrm{~cm}^{3} . \mathrm{s}^{-1}$.

\section{Experiments and protocols}

Two complementary protocols were adopted to measure the threshold of granular erosion, both relying on the equilibrium limit of a particle exposed to the flow. As described in the following paragraphs, the first protocol gives an estimate of the minimal flow condition at which a grain starts moving for the first time while the second one relies on the final scour depth when steady-state equilibrium is eventually reached at the bottom of the eroded crater.

\section{Protocol 1: First grain motion}

This protocol was developed to determine precisely the minimum jet injection condition, i.e. the critical jet velocity, sufficient to initiate the first grain motion at the upper surface of the granular sample. To this end, an acceleration ramp is used to regularly increase the pump flow rate at a rate of approximately $10.5 \mathrm{~cm}^{3} \cdot \mathrm{s}^{-1}$ per minute (minimal value allowed by the pump). This way, and using a synchronized timer, the critical flow rate $Q_{J}{ }^{*}$ and the jet velocity $U_{J}{ }^{*}$ can be measured accurately out of the frame sequence, which was mostly recorded at $1 \mathrm{fps}$. Erosion onset is defined as a grain displacement larger than one particle 


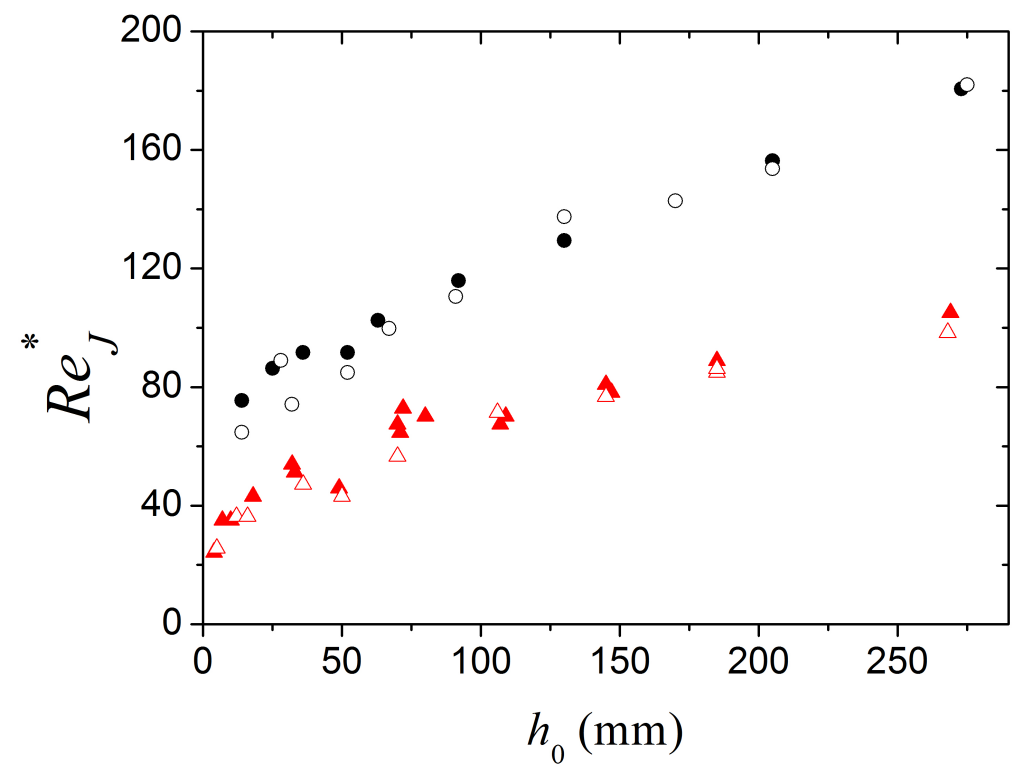

FIG. 3. Critical jet Reynolds number $R e_{J}{ }^{*}$ plotted as a function of $h_{0}$ for beads of diameter $d=1 \mathrm{~mm}$ (red triangles) and $d=5 \mathrm{~mm}$ (black circles) with a jet injection diameter $D_{J}=5 \mathrm{~mm}$ (filled symbols) and $D_{J}=10 \mathrm{~mm}$ (open symbols).

diameter $d$ and that is quickly followed by several other bead movements. In practice, there was no obvious difficulty in stating that erosion has started since these first displacements were much larger than $d$, except for very little cases where a rather isolated and far much anticipated motion (vibration or rolling) of an unusually unstable particle could be observed, but consequently not identified as erosion onset. Several series of such experiments were carried out for different injection diameters $D_{J}\left(D_{J}=5 \mathrm{~mm}\right.$ and $\left.D_{J}=10 \mathrm{~mm}\right)$, different initial distances $h_{0}$ between the injection and the sample top surface $\left(4 \mathrm{~mm}<h_{0}<270 \mathrm{~mm}\right)$, and for all the bead diameters $(d=1,3,5$, and $7 \mathrm{~mm})$. Figure 3 shows the critical jet Reynolds number $R e_{J}{ }^{*}=U_{J}{ }^{*} D_{J} / \nu$ as a function of $h_{0}$ for different configurations. Here it can be noted that the critical jet Reynolds number seems to remain almost the same whatever the injection jet diameter $D_{J}$ for a given couple of initial distance $h_{0}$ and bead diameter $d$. 


\section{Protocol 2: Equilibrium crater depth}

This second protocol made particular use of the transparency of the artificial granular system, which permits to follow directly the evolution of the crater depth at the stagnation point of the impinging jet with the help of the optical techniques described previously in section II A. In the situation where a constant flow rate beyond the previously determined threshold was supplied by the pump, a scour crater would develop featuring a rapid increase of eroded depth $\delta x$ (see definition in Fig. 2) with time. Using almost the same method as described in section II B for the initial bed level, typical evolutions of the scour depth $\delta x$ are presented in Figure 4 for the same jet Reynolds number $R e_{J}$ and initial distance $h_{0}$ but for two different bead diameters and jet diameters. The figure shows that a final equilibrium was quickly reached within a few seconds. Consequently, a similar procedure as in the previous protocol was adopted, introducing the same very slow acceleration ramp $a=10.5 \mathrm{~cm}^{3} . \mathrm{s}^{-1}$ per minute which, in terms of Reynolds number, corresponds to an increase rate of $1.35 \mathrm{~s}^{-1}$ for $D_{J}=5 \mathrm{~mm}$ and $0.68 \mathrm{~s}^{-1}$ for $D_{J}=10 \mathrm{~mm}$, respectively. In this situation, it can be shown that a quasi-steady equilibrium is reached at each time step. Indeed, considering that at the end of the acceleration ramp the Reynolds number is $R e_{J}{ }^{\max }$ and the final scour depth $\delta x^{\max }$, the total duration of scouring during the acceleration ramp reads $\Delta t_{r a m p}=\frac{\pi \nu D_{J}}{4 a}\left(R e_{J}{ }^{\max }-R e_{J}{ }^{*}\right)$. For all of the experiments, $\Delta t_{\text {ramp }}$ remains within the range $100 s<\Delta t_{\text {ramp }}<370$ s. This time is to be compared with the intrinsic scour kinetics observed in Fig.4 where scouring takes about 10s to reach a final crater depth of 50mm (or almost equivalently $6 \mathrm{~s}$ for $25 \mathrm{~mm}$ depth considering $d=5 \mathrm{~mm}$ instead of $d=3 \mathrm{~mm}$ ). On this basis, a typical time of scouring $\Delta t_{\text {scour }}$ can be calculated for each final crater depth $\delta x^{\max }$. For all our data, it is systematically found that $\Delta t_{\text {ramp }} \gg \Delta t_{\text {scour }}$ which validates the quasi-stationary assumption. One gets then a convenient way to measure the equilibrium scour depth $\delta x$ versus the jet Reynolds number $R e_{J}$.

\section{PIV experiments}

To obtain more quantitative results, it was crucial to accurately characterize the free jet between the outlet of the jet injection pipe and the top surface of the bead sample considering that the analytical free jet models constitute a simple and presumably rele- 


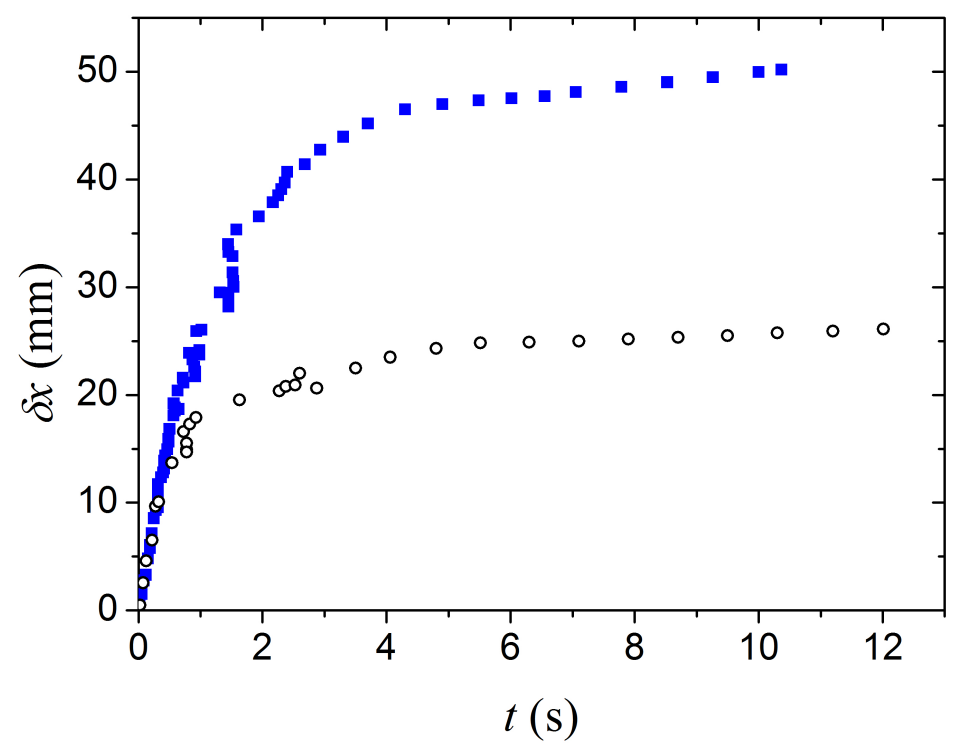

FIG. 4. Crater depth $\delta x$ as a function of time for a sample with bead diameters $d=3 m m$ (blue squares) and $d=5 \mathrm{~mm}$ (black circles) and for two pipe injection diameters $D_{J}$, namely $D_{J}=5 \mathrm{~mm}$ (filled symbols) and $D_{J}=10 \mathrm{~mm}$ (open symbols), with the same jet configuration $\left(h_{0}=70 \mathrm{~mm}\right.$ and $\left.R e_{J}=227\right)$.

vant hydrodynamical reference flow for the impinging jet configuration. A particular effort was placed in getting experimentally the exact parameters of the theoretical laminar free round jet model related to the specific jet conditions tested here (injection tube length and diameter, confinement due to the cell dimensions, density and viscosity properties of the liquid, etc.) instead of merely adopting the available standard values from the literature, with which the former were later compared. A complementary series of experiments was therefore carried out for the acquisition and analysis of the velocity fields of our specific jet in its region of free behavior. For these experiments, the cell was filled only with liquid (no granular sample), while some passive tracers (hollow spheres with diameters in-between 9 and $13 \mu \mathrm{m}$ supplied by Aldrich) were seeded in the liquid. A fast camera (FASTCAM SA3 manufactured by Photron) was then used with a frame rate of 1000 to $1500 \mathrm{fps}$ and a pixel resolution of $512 \times 1024$ (width $\times$ height). From a time averaging on each pair of sequential images, a steady-state velocity field in the laser plane was recovered with a classical Particle Image Velocimetry calculation using the freeware DPIVsoft [33] with an interrogation box dimension $8 \times 32$ (width $\times$ height) in pixels. The related space resolution is about $0.05 \mathrm{~mm}$ 

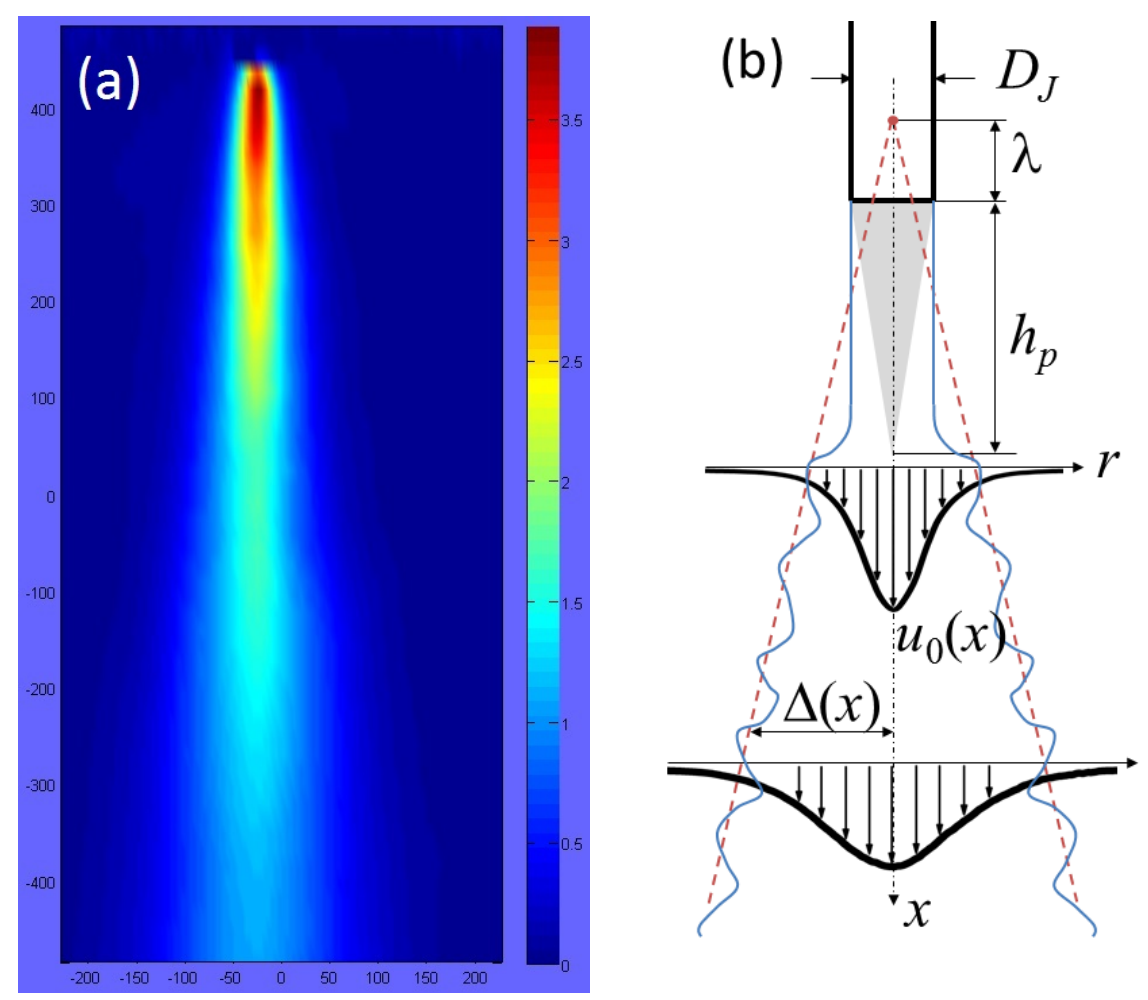

FIG. 5. (a) Typical velocity field of a jet with an initial velocity $U_{J}=0.5 m \cdot s^{-1}$ and a jet injection diameter $D_{J}=5 \mathrm{~mm}$. (b) Sketch of a round immersed free jet with a virtual origin located upward the exit of the injection nozzle, at a distance noted $\lambda$. The length of the potential core is $h_{p}$.

in the radial direction and $0.2 \mathrm{~mm}$ in the vertical direction. A typical stationary velocity field is shown in Figure 5(a) for a jet velocity $U_{J}=0.5 \mathrm{~m} \cdot \mathrm{s}^{-1}$ and a diameter of injection $D_{J}=5 \mathrm{~mm}$.

\section{FREE JET: SELF-SIMILAR MODEL}

As previously mentioned, there is no simple theoretical prediction for a fluid jet impinging on a plane wall, and even less when a crater has been already scoured in the granular sample. However, a straightforward reference flow is the situation of a free jet, for which robust analytical models can be used. This also explains why the free jet model is almost always used in the literature to propose characteristic hydrodynamical quantities. The theoretical framework employed in the present study is based on the self-similar laminar free round jet model, derived by Schlichting [41] from boundary layer approaches and under the assumption that the momentum flux $M$ along the jet remains constant. In this model, considering a 
vertical jet in the downward direction $x$, the vertical component $u$ of the fluid velocity at any position $x$ and radial distance $r$ from the jet centerline reads:

$$
u(x, r)=u_{0}(x) f(\epsilon)
$$

In this expression, $u_{0}(x)$ is the maximal velocity along the jet axis, $\epsilon=r / \Delta(x)$ is the self-similar variable, and $\Delta(x)$ is the characteristic width of the jet. For a round laminar axi-symmetric jet, the function $f$ can be calculated analytically [41], giving:

$$
f(\epsilon)=\left(1+\frac{\epsilon^{2}}{4}\right)^{-2}
$$

The pertinence of the self-similar approach was fairly confirmed by the PIV results as illustrated in Figure 6 where a nice collapse of all the experimental velocity profiles was recovered when plotting $u / u_{0}$ as a function of $r / r_{1 / 2}$ where $u_{0}$ and $r_{1 / 2}$ were the experimental values measured at each position $x$ for the maximal velocity and the half-radius of the jet defined by $u\left(x, r_{1 / 2}\right)=u_{0}(x) / 2$. The data are also in very good agreement with the theoretical prediction which is derived from Eq. 2 and reads:

$$
u(x, r)=u_{0}(x)\left[1+(\sqrt{2}-1)\left(\frac{r}{r_{1 / 2}}\right)^{2}\right]^{-2}
$$

Additionally, the maximal velocity $u_{0}$ and either the jet half-radius $r_{1 / 2}$ or the characteristic jet width $\Delta$ can be all three expressed analytically as a function of $x$, provided that a virtual origin of the jet is considered. As sketched in Figure 5(b), this virtual origin, which is generally located upstream of the nozzle, can be viewed as an extrapolation of the free jet zone in the zone close to the nozzle where a potential flow is still observed. The distance between the nozzle and this virtual origin is denoted $\lambda(\lambda$ being positive when positioned upstream of the outlet by convention). The amplitude $h_{p}$ of the potential core corresponds to an establishing length of the boundary layer to the jet centerline. The maximal velocity $u_{0}$ and the characteristic jet width $\Delta$ far enough from the nozzle, namely for $x>h_{p}$, are given respectively as functions of the distance $x+\lambda$ along the jet from the virtual origin by $[4,40,41]$ :

$$
\begin{gathered}
u_{0}=\frac{3}{8 \pi} \frac{M}{\rho \nu} \frac{1}{x+\lambda} \\
\Delta(x)=4 \nu\left(\frac{\pi}{3} \frac{\rho}{M}\right)^{1 / 2}(x+\lambda)
\end{gathered}
$$




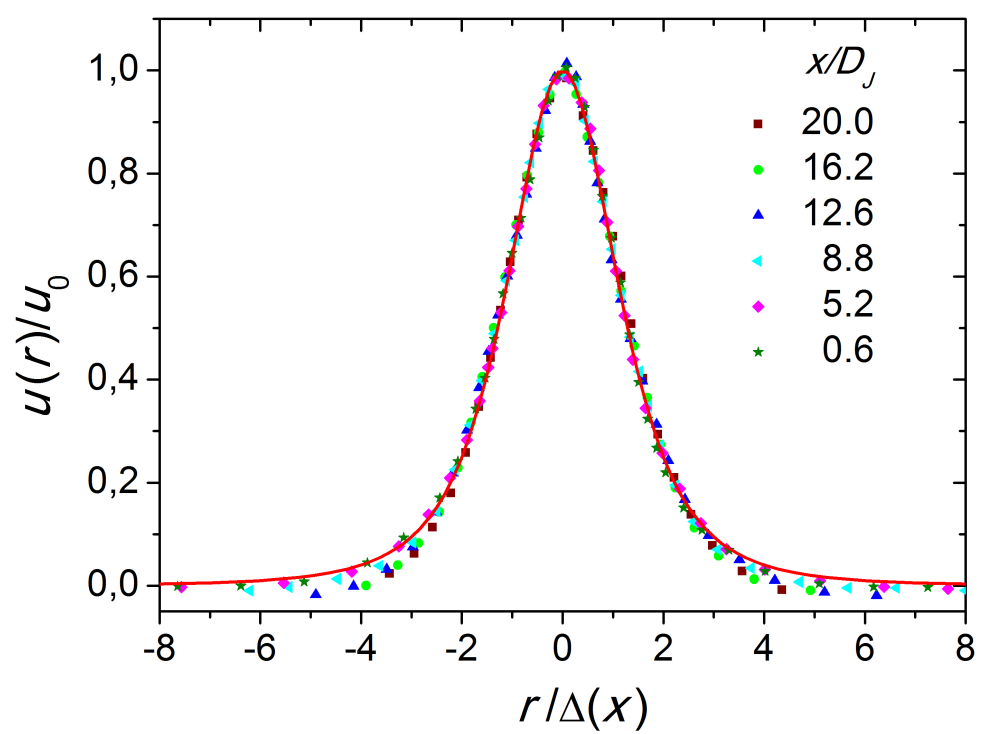

FIG. 6. Collapse of a set of normalized velocity profiles, $u / u_{0}$ versus $r / r_{1 / 2}$, at different distance $x$ from the injection nozzle for $U_{J}=0.5 \mathrm{~m} . \mathrm{s}^{-1}$ and $D_{J}=5 \mathrm{~mm}$. The solid line stands for the theoretical self-similar prediction in Eq. 3 for a fully developed laminar free round jet.

where $\nu$ is the kinematic viscosity of the fluid and $\rho$ its density.

In the present case of an axi-symmetric round jet, the constant flux momentum $M$ reads:

$$
M=\int_{0}^{+\infty} 2 \pi r \rho u^{2}(r) d r
$$

Dimensionally, the flux momentum $M$ must be proportional to $\rho U_{J}^{2} D_{J}^{2}$ and the exact value of the coefficient depends on the flow condition at the exit of the injection nozzle. Considering a Poiseuille profile of velocity inside the pipe, which is a quite reasonable assumption for our experimental conditions with jet Reynolds numbers smaller than 230, the following expression can be straightforwardly obtained:

$$
\frac{M}{\rho}=\frac{\pi}{3} U_{J}^{2} D_{J}^{2}
$$

Then, the previous expressions of $u_{0}$ and $\Delta$ can be simply expressed in terms of the jet Reynolds number $R e_{J}=U_{J} D_{J} / \nu$ as follows:

$$
\begin{gathered}
u_{0}(x)=U_{J} \frac{R e_{J}}{8} \frac{D_{J}}{x+\lambda} \\
\Delta(x)=\frac{4}{R e_{J}}(x+\lambda)
\end{gathered}
$$


The validity of these expressions has been tested in regard of our experimental results. For this purpose, we first checked the linearity of the relationship between $U_{J} / u_{0}$ and $x / D_{J}$ beyond the potential core, i.e. in the free jet region where $x>h_{p}$. For the more usual case of a turbulent jet flow, $h_{p}$ can vary between $6.3 D_{J}$ and $8.3 D_{J}$ according to the literature $[9,24,37]$ or even less as shown by a recent experimental study using PIV [23]. In the present situation, the jet flow regime remains laminar due to the high viscosity of our RIM liquid. It is then possible to refer to the work by Revuelta et al. [40] where the theoretical expression given in Eq. 8 was compared to a direct numerical integration of Navier-Stokes equations for similar flow conditions $\left(R e_{J} \gg 1\right.$ and laminar regime). With the dimensionless coordinate system used by Revuelta et al., a relative error less than $5 \%$ is observed as soon as their dimensionless distance is larger than approximately 0.15 . This can give a practical way to define the potential core length $h_{p}$ which, with the present notations, reads $h_{p} / D_{J}=0.043 R e_{J}$. Based on this definition, a linear regression was calculated for $x>h_{p}$ and the corresponding slope $\alpha$ was evaluated for each configuration $\left(U_{J}, D_{J}\right)$ as illustrated in Figure 7a. Given this rather arbitrary choice made about the range selected for the linear regression, the impact of a slight shift on $h_{p}$ has been tested and led us to estimate that the relative error bars on $\alpha$ are around 10\%. Finally, $1 / \alpha$ was plotted versus the jet Reynolds number $R e_{J}$ and, as expected, Figure $7 \mathrm{~b}$ shows first that a linear relationship can be obtained and, moreover, that the experimental data of $\alpha$ are in fair agreement with the prediction $\alpha=8 / R e_{J}$ coming from Eq. 8 for almost the whole range of parameters explored: $30<R e_{J}<230$, with $D_{J}=5 \mathrm{~mm}$ and $D_{J}=10 \mathrm{~mm}$.

Although the maximal velocity $u_{0}$ was directly measured from the experimental profiles, the characteristic jet width $\Delta$ was itself obtained by fitting each velocity at position $x$ with the theoretical prediction from Eq. 1 and Eq. 2. Then, as illustrated in Figure 8a, the slope $\beta$ between $\Delta / D_{J}$ and $x / D_{J}$ was estimated with the same procedure as for the slope $\alpha$, i.e. by linear regression beyond the potential core $\left(x>h_{p}\right)$, and considering similar error bars of almost $10 \%$. Here again, as shown in Figure $8 \mathrm{~b}$, a plot of $1 / \beta$ versus $R e_{J}$ shows a clear linear trend in close agreement with the theoretical prediction which reads $\beta=4 / R e_{J}$ from Eq. 9.

Note that a slight deviation from the linear prediction is observed for the two lowest Reynolds number values regarding both $1 / \alpha$ and $1 / \beta$ in Fig.7 and Fig.8, even if less pronounced for $1 / \beta$. Probably the condition that $R e_{J} \gg 1$ (see for instance [40]) becomes 

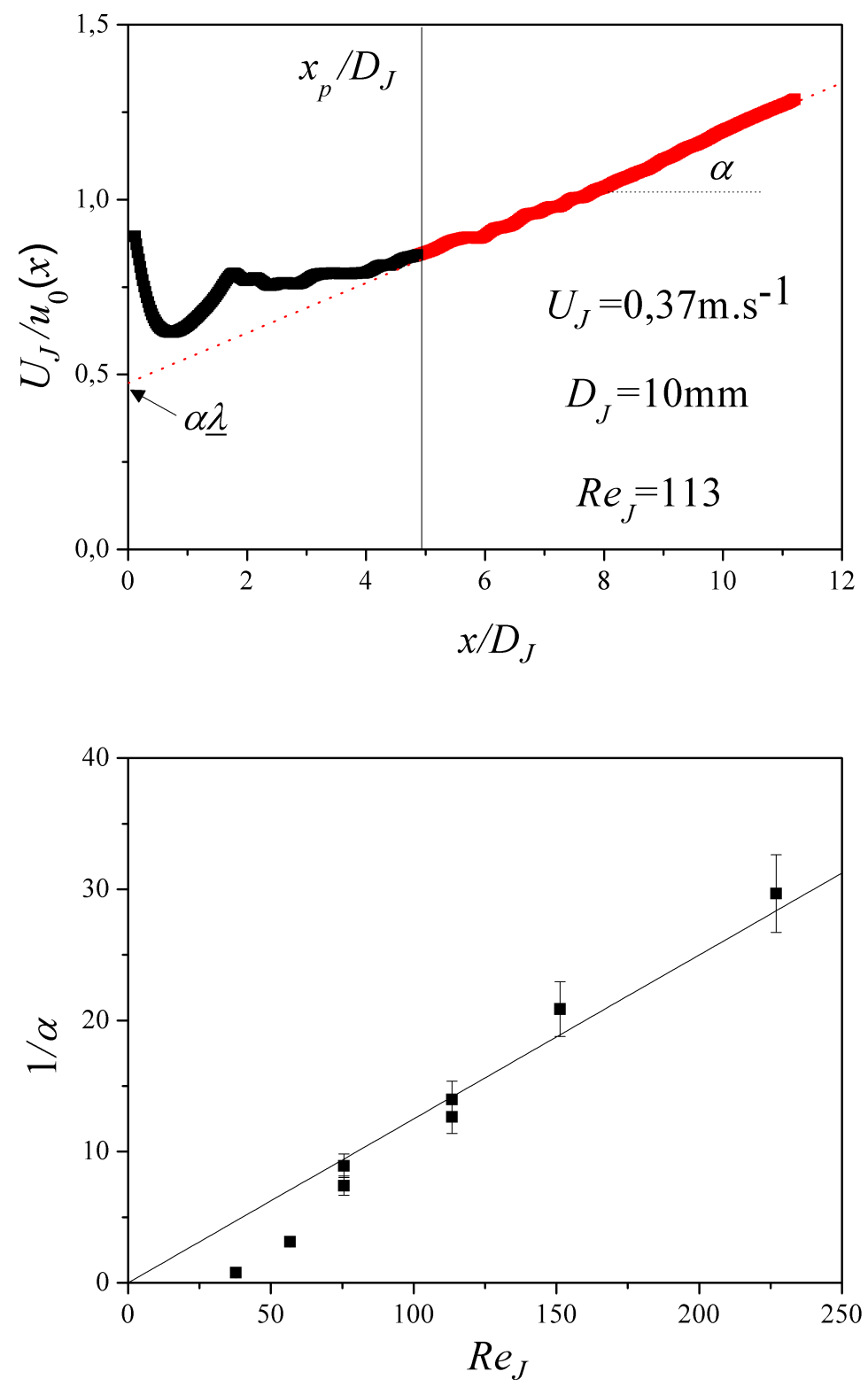

FIG. 7. (Top) Linear relation between $U_{J} / u_{0}$ and $x$ beyond the potential core, namely here for $x>h_{p} \approx 4.9 D_{J}$ (red symbols), with $U_{J}=0.37 \mathrm{~m} \cdot \mathrm{s}^{-1}, D_{J}=10 \mathrm{~mm}$, and $R e_{J}=113$. The slope $\alpha$ and the y-intercept $\alpha \underline{\lambda}$ are sketched on the graph. (Bottom) Inverse values of the slope $\alpha$, obtained from linear regressions between $U_{J} / u_{0}$ and $x / D_{J}$, plotted as a function of the jet Reynolds number $R e_{J}$ for jet configurations $U_{J}=0.25,0.37,0.5$ and $0.75 \mathrm{~m} . \mathrm{s}^{-1}$ with $D_{J}=5$ and $10 \mathrm{~mm}$. The solid line represents the theoretical prediction $1 / \alpha=R e_{J} / 8$ deduced from Eq. 8. 

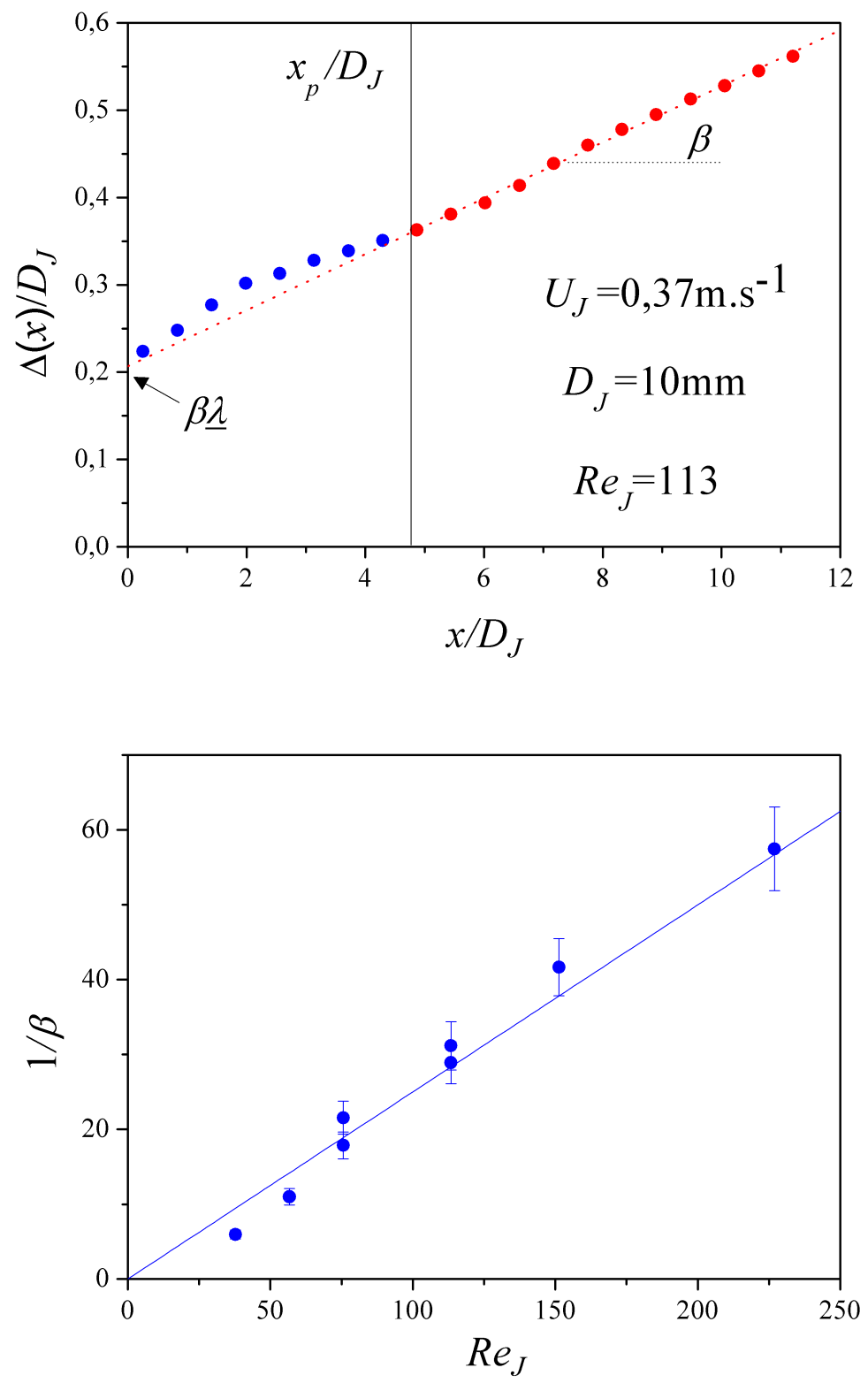

FIG. 8. (Top) Linear relation between $\Delta / D_{J}$ and $x / D_{J}$ beyond the potential core, namely here for $x>h_{p} \approx 4.9 D_{J}$ (red symbols), with $U_{J}=0.37 \mathrm{~m} . \mathrm{s}^{-1}, D_{J}=10 \mathrm{~mm}$, and $R e_{J}=113$. The slope $\beta$ and the $y$-intercept $\beta \underline{\lambda}$ are sketched on the graph. (Bottom) Inverse values of the slope $\beta$, obtained from linear regressions between $\Delta / D_{J}$ and $x / D_{J}$, plotted as a function of the jet Reynolds number for jet configurations $U_{J}=0.25,0.37,0.5$ and $0.75 \mathrm{~m} . \mathrm{s}^{-1}$ with $D_{J}=5$ and $10 \mathrm{~mm}$. The solid line represents the theoretical prediction $1 / \beta=R_{J} / 4$ deduced from Eq. 9. 
questionable.

The last quantity to be determined was the virtual origin length $\lambda$. Experimentally, $\underline{\lambda}=\lambda / D_{J}$ was simply identified from the y-intercepts of the two linear regressions used to estimate the slopes $\alpha$ and $\beta$ respectively (see Fig. 7a and Fig. 8a). This way, two sets of dimensionless values of the virtual origin length $\underline{\lambda}$ were obtained and compared to each other as shown in Figure 9. It can be noted that the results from both methods are reasonably consistent to each other. The error bars, estimated in the same manner as for $\alpha$ and $\beta$, are significantly larger here, around 15 to $20 \%$, and even more in one or two peculiar cases. Furthermore, these experimental data are also reasonably compatible with a theoretical

relation for $\underline{\lambda}$ as a function of the jet Reynolds number $R e_{J}$ for a laminar round jet with a Poiseuille flow condition (i.e. parabolic velocity profile) at injection, coming here again from the comparison between theory and numerical integration made in [40]. With the notations used in the present study, this theoretical prediction reads:

$$
\underline{\lambda}=0.048 R e_{J}
$$

Out of this experimental analysis of the jet generated in our adapted Jet Erosion Test device with an oil mixture approximately thirty times more viscous than water, it can be concluded that the velocity profiles measured by means of the PIV are in remarkable agreement with the theoretical self-similar model of laminar free round jet. Therefore, the theoretical predictions of the model presented in this section as well as the empirical expression of the dimensionless virtual origin length given in Eq. 10 are deemed pertinent and used further in this study.

\section{ANALYSIS}

\section{A. Onset of granular erosion}

The definition of a critical condition for granular erosion requires in the first place to identify the local hydrodynamic forces capable to extract a particle from the top of the granular sample and second to compare them to the forces ensuring the original equilibrium of the particle. Note that some alternative studies have suggested that the governing variable to determine the critical condition for entrainment of a particle by a turbulent flow is an impulse rather than a force [20]. Here, where a laminar flow condition is present, the classical 


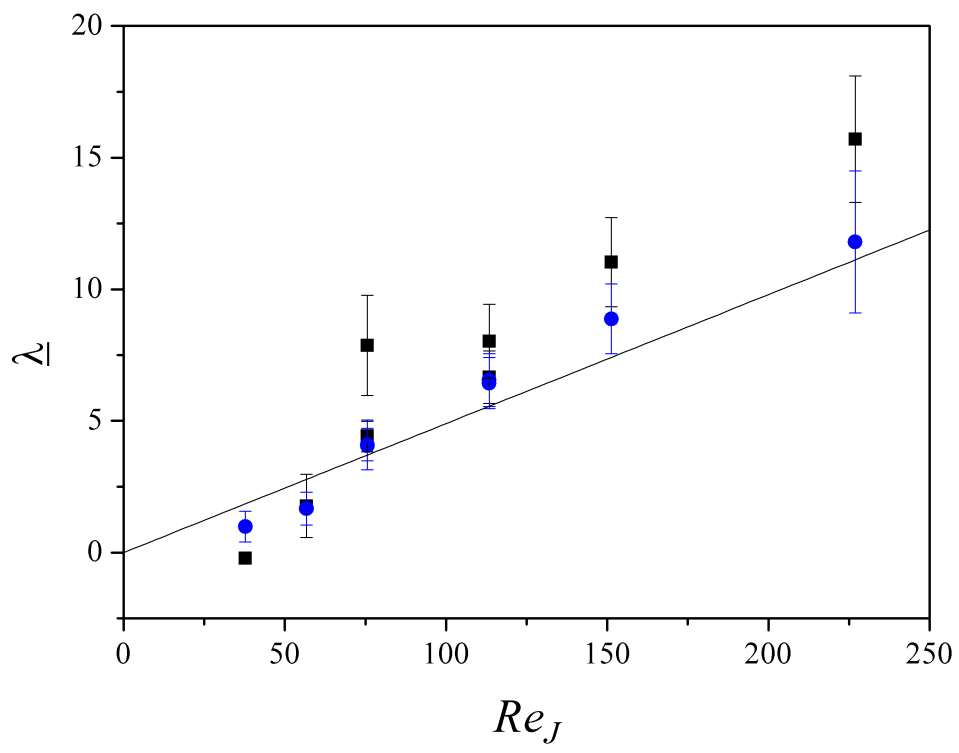

FIG. 9. Dimensionless values $\underline{\lambda}=\lambda / D_{J}$ as a function of the jet Reynolds number $R e_{J}$ obtained from both linear regression methods on $\Delta$ (blue circles) and $U_{J} / u_{0}$ (black squares) as discussed in the text. The solid line stands for Eq. 10.

force approach can be reasonably kept nevertheless. Since there is no cohesion arising from inter-particle interaction, the forces resisting the particle movement include only weight and friction, whose action is directly proportional to weight. As a consequence, a convenient dimensionless quantity for the onset of erosion is the Shields number [15], already briefly defined in the introduction and equal to the ratio of a typical fluid flow force at the surface of the sample $F_{s}$ to the buoyant weight, or equivalently to the ratio of the related hydrodynamic stress $\tau_{s}=F_{s} / d^{2}$ to the buoyant gravitational stress:

$$
S h=\frac{F_{s}}{\left(\rho_{g}-\rho\right) g d^{3}}=\frac{\tau_{s}}{\left(\rho_{g}-\rho\right) g d}
$$

The erosion threshold can consequently be defined as a critical value $S h^{*}$ of the Shields number. Regarding the present situation of an impinging jet on an initially flat granular surface, two local mechanisms are considered here to explain how an individual particle is set in motion by the adjacent fluid flow. The first and rather classical one assumes that the fluid flow around the particle exerts an increasing drag force on the grain until its equilibrium is finally broken as sketched in Figure 10a. As previously stated by Badr et al., one can reasonably expect the corresponding drag stress $\tau_{U}$ to be inertial and consequently 
a)

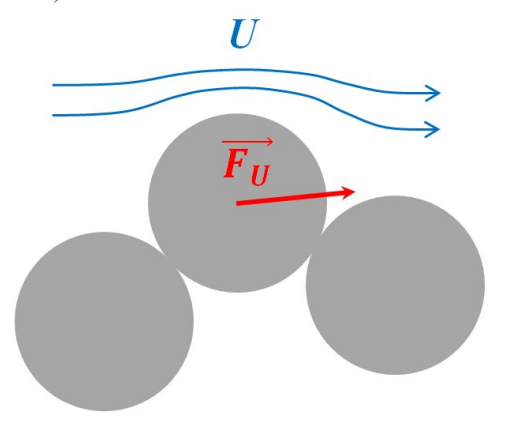

b)

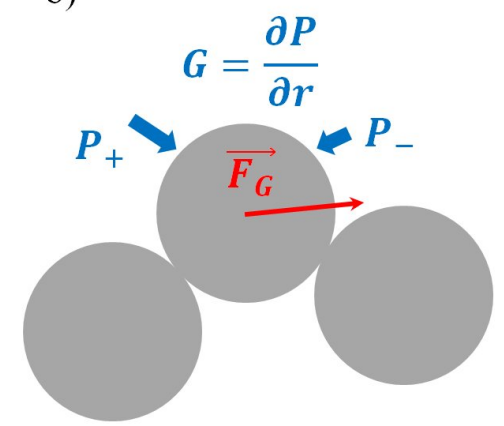

FIG. 10. (a) Inertial drag force $F_{U}$ created by a fluid flow with a characteristic velocity $U$ in the close vicinity of a particle. (b) Inertial pressure force $F_{G}$ created by the difference in dynamical pressure upstream and downstream of a particle, with a characteristic radial gradient of pressure.

simply proportional to $\rho U^{2}$ where $U$ is the mean fluid velocity around the eroded particle. Therefore, determining the maximal value of $\tau_{U}$ and its specific location at the surface $r_{d}$ is equivalent to identifying and locating the extreme value of $U$ at the sample surface. The second mechanism that can be evoked to describe granular erosion is based on the dynamical pressure $P$, and more precisely on its radial gradient $\partial P / \partial r$ which can generate a difference of pressure from one side of the grain to the other as represented schematically in Figure 10b. The resultant pressure force can be directly written as a volume force, function of the local pressure gradient, and finally the related hydrodynamical stress $\tau_{G}$ can be defined as:

$$
\tau_{G}=d \frac{\partial P}{\partial r}
$$

The theoretical location of the first eroded grain coincides therefore with the position of the maximal value of the dynamical pressure gradient exerted by the fluid flow at the upper surface of the granular sample. The main issue is now to define some relevant characteristic extreme values for the identified hydrodynamical quantities involved in the grain erosion, namely the maximal local superficial velocity $U_{\max }$ and the maximal gradient (in absolute value) of the dynamical pressure $G_{\max }=\left|\frac{\partial P}{\partial r}\right|_{\max }$. For this purpose, it seems appropriate to consider firstly the flow configuration of a circular impinging jet on a flat solid wall, which has motivated many studies over the years including notably the following contributions [8, 9, 23, 37]. Here again, all of these works have focused exclusively on turbulent jets, with jet Reynolds numbers being of at least two orders of magnitude larger than in the present situation. Regarding the wall shear stress, several empirical expressions can be 
found as well in the literature, for instance in [8, 23, 37]. Dimensionally, the maximum stress appears to be simply proportional to the square of the centerline velocity $u_{0}$ of a free jet with the same injection Reynolds number and at the same distance from the injection nozzle. It should be noted that in this case the expression for $u_{0}$ is no longer the one shown in Eq. 8 obtained for our laminar free jet. For the case of a turbulent free jet, the different quantitative values for all of the centerline velocity decay rate, the radial expansion, the potential core length and the virtual origin depend more or less heavily on the specific conditions of injection and other boundary conditions [4, 23, 44]. Note also that there is still no consensus in the literature $[8,23,37]$ neither on the position of the maximum shear stress $\tau_{\max }$ nor on the exact value of the coefficient of proportionality between $\tau_{\max }$ and $\rho u_{0}{ }^{2}$, often called coefficient of friction $C_{f}[23,24]$. A typical value of $C_{f}$ is around $4 \times 10^{-3}$ for turbulent flow conditions $[8,23,24]$. Considering now the pressure exerted by the normal jet at the wall, the data are more scarce, where for instance the results presented by Beltaos and Rajaratnam [8] showed a gaussian type distribution. To our knowledge, almost all previous works that have considered the implications for erosion assessment of natural soils, both cohesive or cohesionless, have directly made use of the results from turbulent wall impinging jets (plane or circular) [9, 24-26, 30] without taking account of the significant differences in terms of roughness, permeability [43] and, above all, morphology once the scouring process has been initiated. An even simpler approach was adopted by Badr et al. [5] for the case of granular erosion by both laminar and turbulent plane jets, considering as a strong assumption that, despite the complex flow configuration of a jet impinging on a sediment floor, the relevant shear-stress characteristic value exerted at the upper surface of the granular system was directly related to the maximal velocity predicted by the free jet models. Here we nevertheless decided to follow the same procedure and, referring to the free jet centerline velocity $u_{0}$ at a distance $h_{0}$ to the jet nozzle which is given by Eq. 8 with $x=h_{0}$, the relevant maximal superficial velocity is consequently $U_{\max }=u_{0}\left(h_{0}\right)$ similarly as the one chosen by Badr et al. [5]. Since the kinematic pressure of the free jet at the distance $h_{0}$ reads simply $P=\frac{1}{2} \rho\left[u\left(h_{0}, r\right)\right]^{2}$, the maximal value $G_{\max }$ of the absolute pressure gradient $\left|\frac{\partial P}{\partial r}\right|$ can be expressed in the following manner after some algebraic manipulations:

$$
G_{\max }=\frac{2}{3}\left(\frac{9}{10}\right)^{5} \frac{\rho\left[u_{0}\left(h_{0}\right)\right]^{2}}{\Delta\left(h_{0}\right)}
$$

From there, two alternative Shields numbers can be defined and, omitting prefactors, read 
respectively:

$$
\begin{aligned}
& S h_{U}=\frac{\left[u_{0}\left(h_{0}\right)\right]^{2}}{g^{\prime} d} \\
& S h_{G}=\frac{\left[u_{0}\left(h_{0}\right)\right]^{2}}{g^{\prime} \Delta\left(h_{0}\right)}
\end{aligned}
$$

where $g^{\prime}$ stands for the reduced gravity and is defined by $g^{\prime}=\left(\rho_{g} / \rho-1\right) g$.

For a comparison, it can be noted that the definition of $S h_{U}$ shown here is the same as the one used by Badr et al. [5]. According to Eq. 8 and Eq. 9, the hypothesis that there is a constant critical Shields number, either $S h_{U}{ }^{*}$ or $S h_{G}{ }^{*}$, , for any given particle diameter leads, after some manipulations, to the following relationships between the critical jet Reynolds numbers $R e_{J}{ }^{*}$ determined in our experiments with the first protocol and the distance $h_{0}$ from the nozzle to the sample surface:

$$
\begin{aligned}
R e_{J}{ }^{* 2} & =\left(64 S h_{U}{ }^{*} A r\right)^{1 / 2} \frac{h_{0}+\lambda}{d} \\
R e_{J}{ }^{* 5 / 3} & =\left(256 S h_{G}{ }^{*} A r\right)^{1 / 3} \frac{h_{0}+\lambda}{d}
\end{aligned}
$$

In these equations, we have introduced the Archimedes number $A r$ which is defined as follows:

$$
A r=\frac{g^{\prime} d^{3}}{\nu^{2}}
$$

Then, these relationships have been compared to our experimental data for each series corresponding to a particle diameter $d$, whatever the injection diameter $D_{J}$. As shown in the examples on Figure 11, a rather nice agreement is observed almost equivalently in both cases.

Each linear regression can be subsequently used to extract the corresponding value of either $S h_{U}{ }^{*}$ or $S h_{G}{ }^{*}$ for each particle diameter (or equivalently for each value of $A r$ ). It appears interesting to see these values plotted in terms of the particle Reynolds number $R e_{p}=\frac{u_{0} d}{\nu}$ as shown in Figure 12. From Figure 12, it is straightforward to appreciate that an almost constant value of $S h_{U}{ }^{*}$ can be derived while $S h_{G}{ }^{*}$ increases continuously with $R e_{p}$. This result suggests that $S h_{U}$ appears as a better choice than $S h_{G}$ to account for granular erosion by an impinging jet. Note also that the value obtained for $S h_{U}{ }^{*}$, namely $S h_{U}{ }^{*}=1.88 \pm 0.54$, is remarkably consistent with the results of Badr et al. [5], which were based on the erosion induced by laminar to turbulent plane jets showing $S h_{U}{ }^{*}=1.2 \pm 0.6$ for particle Reynolds in an almost similar range than in the present study: $3 \lesssim R e_{p} \lesssim 100$. 

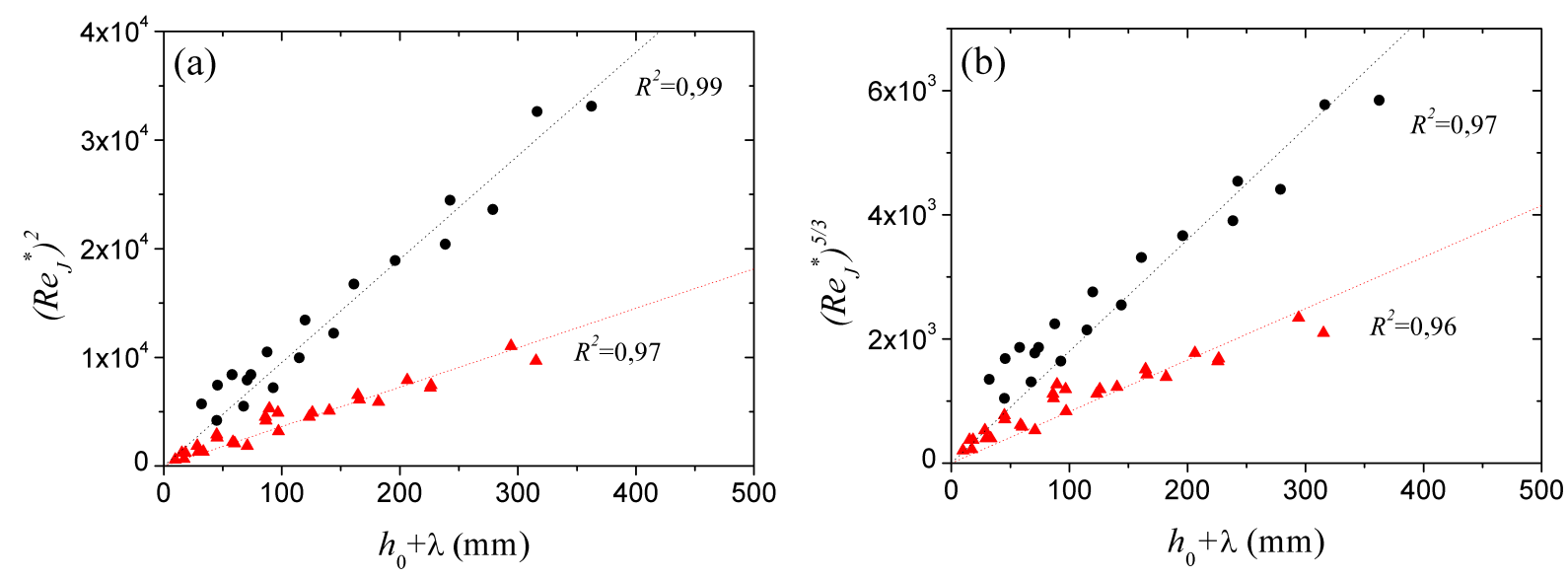

FIG. 11. Plot of (a) $R e_{J}{ }^{2}$ and (b) $R e_{J}{ }^{* 5 / 3}$ as a function of the total jet height $h_{0}+\lambda$ for two bead diameters: $d=1 \mathrm{~mm}$ (red triangles) and $d=5 \mathrm{~mm}$ (black circles). The dotted lines are linear fits with zero intercept.

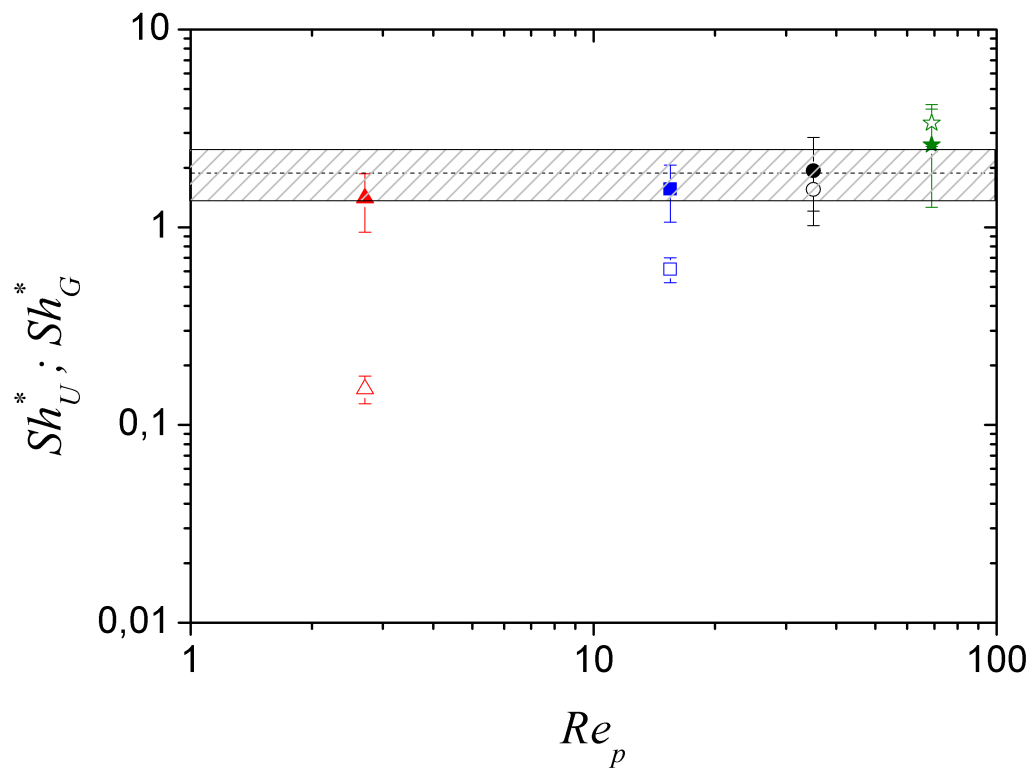

FIG. 12. Critical values $S h_{U}{ }^{*}$ (filled symbols) and $S h_{G}{ }^{*}$ (open symbols) of the inertial Shields numbers, built on the drag force and the pressure gradient respectively, plotted versus the particle Reynolds number $R e_{p}$ for the four different bead diameters: $d=1 \mathrm{~mm}$ (red triangles), $d=3 \mathrm{~mm}$ (blue squares), $d=5 \mathrm{~mm}$ (black circles), and $d=7 \mathrm{~mm}$ (green diamonds). The mean value of $S h_{U}{ }^{*}$ is 1.88 (dotted line) with an error bar of 0.54 (shaded area). 
Author-produced version of the article published in Physical Review Fluids, 2017, 2, 034302.

The original publication is available at https://journals.aps.org/prfluids/abstract/10.1103/PhysRevFluids.2.034302 DOI: 10.1103/PhysRevFluids.2.034302

\section{B. Tentative crater jet model inside the granular medium}

From the previous analysis of a jet impinging on a flat granular surface, it can so far be inferred that the first particle motion is approximately ruled by a constant value of the inertial Shields number $S h_{U}{ }^{*}$ defined from an inertial drag force. For a given grain diameter, this criterion is equivalent to a critical value of the local flow velocity at the sample surface. Considering now the situation where this critical condition is exceeded, giving rise to the appearance and development of a scour crater at the apex of the impinging jet, a considerable evolution of the flow behavior can be expected. In fact, the lateral part of the jet is still deviated radially at the vicinity of the sample top surface but another increasing part penetrates inside the crater generating a recirculating flow and a consequent increase in energy dissipation. In order to quantify the effect of this crater vortex dissipation, the experimental results obtained with our second protocol (see section II C) can be suitably analyzed as explained hereafter. This protocol was specifically chosen to reach a quasi-steady equilibrium state where erosion definitely ceases at the bottom of the crater. In principle, it appears reasonable to consider that the condition for the erosion stop is the same than for the erosion start, or in other words, that the local flow velocity in the floor area of the scour hole is exactly equal to the critical velocity given by the critical Shields number $S h_{U}{ }^{*}$. Under this assumption, the maximal velocity in a free jet $u_{0}$ at the same distance $h_{0}+\delta x$ from the injection nozzle can be directly compared to the maximal local flow velocity at the bottom of the crater denoted $u_{0}^{c}$ which is equal to the critical erosion velocity in the specific situation corresponding to this second protocol. Let us this way define the ratio $C$ between $u_{0}^{c}$ and $u_{0}$ :

$$
u_{0}^{c}=C \cdot u_{0}
$$

By definition $C=1$ for a flat granular surface (first grain motion) and $C<1$ when a scour crater exists, with a value of $C$ decreasing progressively with an increase of the crater depth $\delta x$. A convenient way to calculate the coefficient $C$ with our experimental protocol consists in determining the critical jet velocity $U_{J}{ }^{*}$ for which the first grain motion is detected at the initially flat sample surface and then, at each jet velocity $U_{J}$ above this threshold, to measure directly from the image sequences the quasi-equilibrium crater depth $\delta x$ inside the index-matched system with the optical techniques mentioned before. From the self-similar model of the free round jet, and more precisely relying on Eq. 8, $C$ can be written 


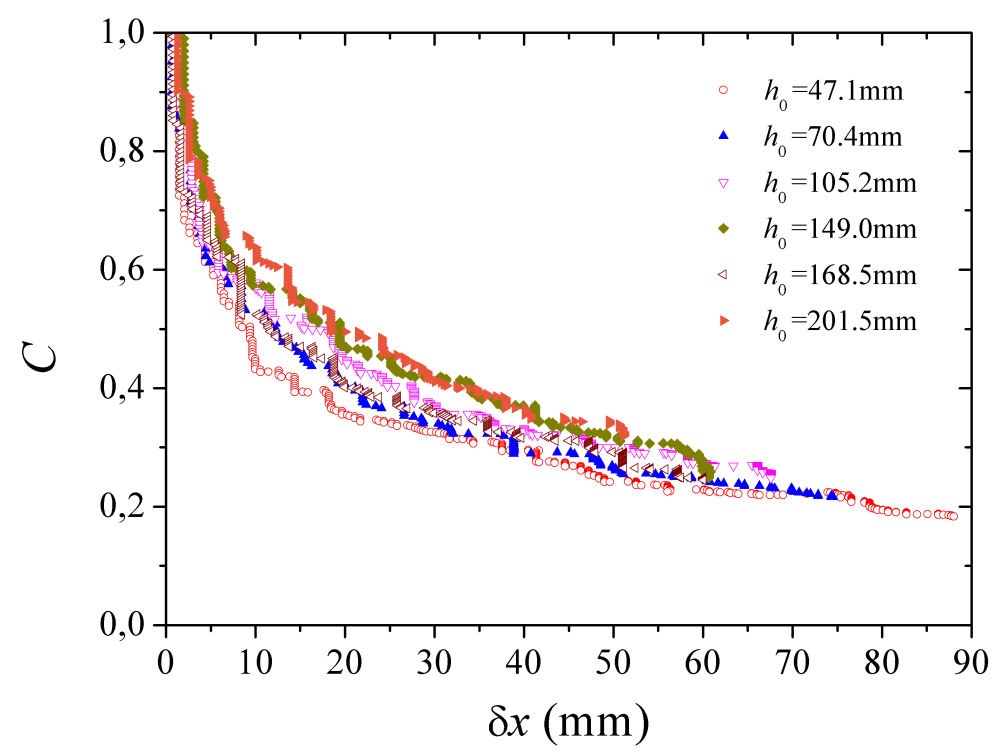

FIG. 13. Values of the proportionality coefficient $C$ as a function of $\delta x$ obtained for beads of diameter $3 \mathrm{~mm}$ and for different initial distances $h_{0}$ from the jet nozzle $\left(D_{J}=5 \mathrm{~mm}\right)$ to the sample surface.

as follows where $U_{J}$ is the jet velocity, $\delta x$ the crater depth, $\lambda$ the virtual origin and where the exponent $*$ stands for the critical values at the initial bed surface (i.e. at a distance $h_{0}$ from the injection nozzle):

$$
C=\left(\frac{U_{J}^{*}}{U_{J}}\right)^{2}\left(\frac{h_{0}+\delta x+\lambda}{h_{0}+\lambda^{*}}\right)
$$

Figure 13 shows the evolution of the coefficient $C$ obtained in this way as a function of $\delta x$ for a sample with bead diameter $d=3 \mathrm{~mm}$, injection diameter $D_{J}=5 \mathrm{~mm}$, and for different values of $h_{0}$. Note that the distance $\lambda$ to the virtual origin was calculated from Eq. 10 .

All the curves relax following a similar shape which suggests the existence of a characteristic flow attenuation depth within the crater which increases with the initial distance $h_{0}$. This length appears to be most probably related to the dimensions of the crater. Under the reasonable assumption that the width of the crater is directly correlated to the jet's width $\Delta\left(h_{0}\right)$, referring again to the situation of a laminar free jet as previously, $\Delta\left(h_{0}\right)$ could be a good candidate for the quantification of this velocity decay length due to a flow attenuation in the crater. To test quantitatively this hypothesis, the same curves presented in Figure 13 have been plotted now against $\frac{\delta x}{\Delta\left(h_{0}\right)}$ in Figure 14. As it can be seen, the different curves 


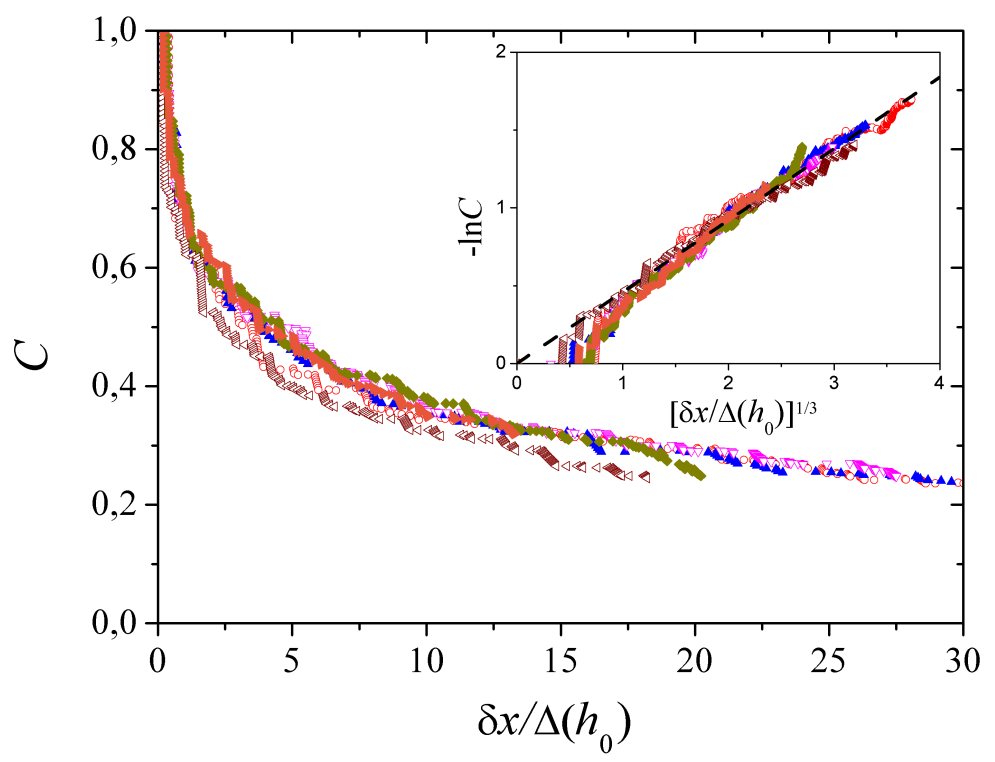

FIG. 14. Values of the proportionality coefficient $C$ as a function of $\frac{\delta x}{\Delta\left(h_{0}\right)}$ obtained for beads of diameter $3 \mathrm{~mm}$ and for different initial distances $h_{0}$ from the jet nozzle $\left(D_{J}=5 \mathrm{~mm}\right)$ to the sample surface (same symbol conventions as in Figure 13). Inset: Plot of $-\ln (C)$ versus $\left[\frac{\delta x}{\Delta\left(h_{0}\right)}\right]^{1 / 3}$. The dashed solid line stands for the empirical law given in Eq. 21 with $k=0.45$.

collapse nicely on a unique trend confirming the pertinence of the assumption. Note also that the following empirical law can be proposed for $C$ with acceptable accuracy:

$$
C=\exp \left(-k\left[\frac{\delta x}{\Delta\left(h_{0}\right)}\right]^{1 / 3}\right)
$$

with $k \sim 0.45$. The latter result is less obvious when considering the other injection diameter $D_{J}=10 \mathrm{~mm}$, suggesting however a larger value for $k(k \sim 0.7)$. Nonetheless, it was not possible to conduct satisfactorily the same analysis for another set of data obtained with larger beads of diameter $d=5 \mathrm{~mm}$, probably due in large part to finite size effects which give rise to strongly intermittent time evolutions of the crater depth featuring successive jumps and plateaus. Therefore, it appears important to remain at this stage cautious on the role of $\Delta\left(h_{0}\right)$ to account for the flow attenuation within the scouring crater. The same holds for the use of the empirical law in Eq. 21, which needs to be confirmed and possibly improved or amended. Further investigations are therefore necessary in order to be able to reach a definitive conclusion on this point. 


\section{CONCLUSION}

This paper presents several experimental techniques to analyse the erosion process of an artificial granular material subjected to the Jet Erosion Test. The specific hydrodynamic conditions of the immersed jet have been analyzed, validating the use of the theoretical framework of a self-similar jet for the studied cases (jet Reynolds number between 30 and 230). The onset of erosion for the cohesionless granular bed was precisely captured with an analysis of sequential images of the sample, which led to the identification of the best candidate of a dimensionless number to characterize the erosion resistance of a granular material. The results presented here support the proposal of a critical Shields number based on the jet velocity, rather than on the pressure gradient, which can be expressed in terms of the particular Reynolds number. It should be noted that our results are remarkably similar to the previous ones obtained by Badr et al. [5] for a plane jet. Finally, the paper presents a tentative empirical expression to model the jet velocity decay inside the scouring crater taking into account the erosion kinetics. Despite its limitations, the method proposed here shows the importance of the distinction between the length of the free jet from the nozzle to the surface of the sample and that of the crater developing as the jet erosion proceeds.

\section{ACKNOWLEDGMENTS}

This work benefits from fruitful discussions with P. Aussillous, J.-Y. Delenne, G. Gauthier, P. Gondret, and Y. Khidas. The authors acknowledge financial support from the region PACA (Provence-Alpes-Côte d'Azur, France) and valuable exchanges with D. Chaussée (Suez Consulting Corp.). The authors also thank two anonymous reviewers for thoughtful comments that improved the present manuscript.

[1] O. O. Aderibigbe and N. Rajaratnam. Erosion of loose beds by submerged circular impinging vertical turbulent jets. J. Hydraul. Res. 34(1), 19-33 (1996).

[2] J. R. Agudo and A. Wierschem. Incipient motion of a single particle on reguler substrates in laminar shear flow. Phys. Fluids 24, 093302, (2012). 
[3] R. Ariathurai and K. Arulanandan. Erosion rates of cohesive soils. J. Hydraul. Div. 104(2), 279-283 (1978).

[4] S. Badr. Erosion d'un milieu granulaire par un jet. PhD thesis, Université Paris-Sud (2014).

[5] S. Badr, G. Gauthier and P. Gondret. Erosion threshold of a liquid immersed granular bed by an impinging plane liquid jet. Phys. Fluids 26, 023302 (2014).

[6] S. Badr, G. Gauthier and P. Gondret. Crater jet morphology. Phys. Fluids 28, 033305, (2016).

[7] R. Béguin, P. Philippe and Y.-H. Faure. Pore-Scale Flow Measurements at the Interface between a Sandy Layer and a Model Porous Medium: Application to Statistical Modeling of Contact Erosion. J. Hydraul. Eng. 139(1), 1-11 (2013).

[8] S. Beltaos and N. Rajaratnam. Impinging circular turbulent jets. J. Hydraul. Div. 100, 13131328 (1974).

[9] S. Beltaos and N. Rajaratnam. Impingment of axisymmetric developing jets. J. Hydraul. Res. 15(4), 311-326 (1977).

[10] N. Benahmed and S. Bonelli. Investigating concentrated leak erosion behaviour of cohesive soils by performing hole erosion tests. European Journal of Environmental and Civil Engineering 16(1), 43-58 (2012).

[11] B. J. Boersma, G. Brethouwer and F. T. M. Nieuwstadt. A numerical investigation on the effect of the inflow conditions on the selfsimilar region of a round. Phys. Fluids 10, 899-909, (1998).

[12] S. Bonelli (Ed.). Erosion of geomaterials. Wiley-ISTE (2012).

[13] S. Bonelli (Ed.). Erosion in Geomechanics Applied to Dams and Levees. Wiley-ISTE (2013).

[14] J. L. Briaud, F. C. K. Ting, H. C. Chen, Y. Cao, W. Han and K. W. Kwak. Erosion Function Apparatus for scour rate predictions. J. Geotech. Geoenviron. Eng. 127(2), 105-113 (2001).

[15] J. M. Buffington. The legend of A. F. Shields. J. Hydraul. Eng. 125(4), 376-387 (1999).

[16] F. Charru, H. Mouilleron and O. Eiffel. Erosion and deposition of particles on a bed sheared by a viscous flow. J. Fluid Mech. 519, 55-80 (2004).

[17] A. H. Clark and R. P. Berhinger. Jet-induced 2-D crater formation with horizontal symmetry breaking. Granular Matter 16(4), 433-440 (2014).

[18] J. J. Derksen. Simulations of granular bed erosion due to laminar shear flow near the critical Shields number. Phys. Fluids 23, 113303, (2011). 
[19] J. A. Dijksman, F. Rietz, K. A. Lörincz, M. van Hecke and W. Losert. Refractive index matched scanning of dense granular materials. Rev. Sci. Instrum. 83, 011301 (2012).

[20] P. Diplas, C. L. Dancey, A. O. Celik, M. Valyrakis, K. Greer and T. Akar. The Role of Impulse on the Initiation of Particle Movement under Turbulent Flow Conditions. Science 322(5902), 717-720 (2008).

[21] P. F. Dufois, R. Verney, P. Le Hir and F. Dumas. Impact of winter storms on sediment erosion in the Rhon River prodelta and fate of sediment in the Gulf of Lions (North Western Mediterranean Sea. Continental Shelf Research 72, 57-72 (2014).

[22] M. Foster, R. Fell and M. Spannagle. The statistics of embankment dam failures and accidents. Can. Geotech. J. 37, 1000-1024 (2000).

[23] S. M. Ghaneeizad and J. F. Atkinson. Effect of flow confinement on the hydrodynamics of circular impinging jets: implications for erosion assessment. Environ. Fluid Mech. 15, 1-25 (2015).

[24] G. J. Hanson and K. R. Cook. Apparatus, Test Procedures, and Analytical Methods to Measure Soil Erodibility In Situ. Applied Engineering in Agriculture 20(4), 455-462 (2004).

[25] A. J. Hogg, H. E. Huppert and W. B. Dade. Erosion by planar turbulent wall jets. J. Fluid Mech. 338, 317-340 (1997).

[26] H. Kobust, P. Leister and B. Westrich. Flow field and scouring effects of steady and pulsating jets impinging on a movable bed. J. Hydraul. Res. 17(3), 175-192 (1979).

[27] S. B. Kuang, C. Q. LaMarche, J. S. Curtis and A. B. Yu. Discrete particle simulation of jet-induced cratering of a granular bed. Powder Technology 239, 319-336 (2013)

[28] C. Q. LaMarche and J. S. Curtis. Cratering of a particle bed by a subsonic turbulent jet: Effect of particle shape, size and density. Chemical Eng. Science 138, 432-445 (2015)

[29] A. E. Lobkovsky, A. V. Orpe, R. Molloy, A. Kudrolli and D. H. Rothman. Erosion of a granular bed driven by laminar fluid flow. J. Fluid Mech. 605, 47-58 (2008).

[30] K. A. Mazurek and T. Hossain. Scour by jets in cohesionless and cohesive soils. Can. J. of Civil Eng. 34, 744-751 (2007).

[31] F. Mercier, S. Bonelli, P. Pinettes, F. Golay, F. Anselmet and P. Philippe, Comparison of Computational Fluid Dynamic Simulations with Experimental Jet Erosion Tests Results. J. Hydraul. Eng. 140(5), 04014006 (2014). 
[32] P. T. Metzger, C. D. Immer, C. M. Donahue, B. M. Vu, R. C. Latta III and M. Deyo-Svendsen. Jet-induced cratering of a granular surface with application to lunar spaceports. J. Aerosp. Eng. 22(1), 24-32 (2009).

[33] P. Meunier and T. Leweke. Analysis and treatment of errors due to high velocity gradients in particle image velocimetry. Experiments in Fluids 35(5), 408-421 (2003).

[34] M. Ouriemi, P. Aussillous, M. Medale, Y. Peysson and E. Guazzelli. Determination of the critical Shields numlber for particle erosion in laminar flow. Phys. Fluids 19, 061706, (2007).

[35] T. M. Parchure and A. J. Mehta, Erosion of Soft Cohesive Sediment Deposits. J. Hydraul. Eng. 111(10), 1308-1326 (1985).

[36] E. Partheniades, Erosion and deposition of cohesive soil. J. hydraul. division 91(1), 105-139 (1965).

[37] D. Phares, G. T. Smeley and R. C. Flagan, The wall shear stress produced by the normal impingement of a jet on a flat surface. J. Fluid Mech. 418, 351-375 (2000).

[38] P. Philippe and M. Badiane, Localized fluidization in a granular medium. Physical Review E 87, 042206 (2013).

[39] K. S. Pouv, A. Besq, S. S. Guillou, E. A. Toorman, On cohesive sediment erosion: A first experimental study of the local processes using transparent model materials. Advances in Water Resources 72, 71-83 (2014).

[40] A. Revuelta, A. L. Sánchez and A Liñán, The virtual origin as a first-order correction for the far-field description of laminar jets. Phys. Fluids 14(6), 1821-1824 (2002).

[41] H. Schlichting, Boundary layer theory. McGraw Hill Book Co., New York (1960).

[42] B. R. Sutherland and S. Dalziel, Bedload transport by a vertical jet impinging upon sediments. Phys. Fluids 26(3), 035103 (2014).

[43] S. Webb and I. Castro. Axisymmetric jets impinging on porous walls. Experiments in Fluids 40, 951-961 (2006).

[44] G. Xu and R. A. Antonia. Effect of different initial conditions on a turbulent round free jet. Experiments in Fluids 33, 677-683 (2002). 